\title{
Reducing vehicle interior NVH by means of locally resonant metamaterial patches on rear shock towers
}

\author{
Author, co-author (Do NOT enter this information. It will be pulled from participant tab in \\ MyTechZone) \\ Affiliation (Do NOT enter this information. It will be pulled from participant tab in MyTechZone)
}

\begin{abstract}
Stringent regulations for $\mathrm{CO}_{2}$ emissions and noise pollution reduction demand lighter and improved Noise, Vibration Harshness (NVH) solutions in automotive industries. Designing light, compact and, at the same time, improved NVH solutions is often a challenge, as low noise and vibration levels often require heavy and bulky additions, especially to be effective in the low frequency regime. Recently, locally resonant metamaterials have emerged among the novel NVH solutions because of their performant $\mathrm{NVH}$ properties combined with lightweight and compact design. Due to the characteristic of stop band behavior, frequency ranges where free wave propagation is inhibited, metamaterials can beat the mass law, be it at least in some tunable frequency ranges. Previously the authors demonstrated how metamaterials can reduce the vibrations in a simplified shock tower upon shaker excitation. In this work, the authors apply the metamaterial concept on the real rear shock towers of a vehicle. In order to be able to benchmark the solution, a test vehicle is chosen, which is equipped in its commercial version with a $1.46 \mathrm{~kg}$ tuned vibration absorber (TVA) on each of the rear shock towers as $\mathrm{NVH}$ solution. It is shown that the metamaterial solution allows to achieve similar interior NVH performance, while reducing the added mass by $48 \%$. The metamaterial additions are realized through additive manufacturing and they are designed to be effective around $190 \mathrm{~Hz}$, as was the case for the original solution. Both experimental results and numerical validation of a road test are presented.
\end{abstract}

\section{Introduction}

In the last decades, stringent environmental regulations and economical requirements have influenced the design of the vibroacoustic solutions in industry, in particular in the automotive sector. Lightweight design is required to reduce $\mathrm{CO}_{2}$ emissions [1]. At the same time, the awareness of noise pollution on human health [2] demands enhanced NVH solutions. Combining lightweight and performant $\mathrm{NVH}$ solutions is often a challenge, since the $\mathrm{NVH}$ performance that a partition can achieve scales with its own mass [3].

Locally resonant metamaterials recently have emerged among the common $\mathrm{NVH}$ solutions to cope with this challenging task. By embedding resonant elements into a host structure, a material structure can be obtained, which shows stop band behavior in tunable frequency ranges, related to the natural frequency of the added resonant elements [4]. In these stop bands, no structural waves can freely propagate into the system, hence vibrations are attenuated, resulting in reduction of

Page 1 of 9 acoustic radiation and sound transmission [5, 6, 7]. Therefore, retaining low mass and low volume, locally resonant metamaterials can outperform the common NVH solutions, especially at the low frequency range.

In previous publications, the authors showed how metamaterial solutions can isolate structural vibrations along a transfer path [8]. Numerical and experimental results on a simplified shock tower of a vehicle [9] indicated the potential for vibration reduction without the need to treat the whole host structure, as long as resonant elements are added on all the relevant transfer paths. In this work, the metamaterial concept is applied on shock towers of a commercial vehicle. The objective is to reduce the acoustic pressure levels in the interior compartment caused by vibrational energy, generated by tire/road interaction while driving and entering in the vehicle body through the rear suspension assembly system $[10,11,12]$. The vehicle is equipped with a TVA on each of the rear shock towers to reduce acoustic pressure levels in the vehicle in a frequency region around $190 \mathrm{~Hz}$ and, therefore, this can serve as a benchmark for the metamaterial solution. The metamaterial solution consists of patches, added on the area surrounding the connection of the shock absorber with the shock tower.

This paper is organized as follows: after the introduction, the application case is presented in section 2 , followed by the description of the design of the metamaterial solution in section 3 . The test procedure is described in Section 4. Test results are presented and discussed in Section 5. Finally, the findings are summarized in the conclusions.

\section{Application case}

Low frequency structure borne noise in the interior compartment of vehicles is in large part caused by tire/road interaction while driving. This interaction generates vibrational energy, which is injected inside the vehicle body through the suspensions $[10,11]$. This can result in vehicle body parts vibrating and radiating noise. The types of suspension system characterize the mechanical transmission paths, hence the frequency range where the maximum vibrational energy is injected inside the vehicle body.

A European SUV is chosen as an application case. It has a MacPherson Strut as rear suspension and tires model 235/60 R18. Because of this type of suspension assembly system, vibrational energy is injected inside the vehicle body in a frequency range around $190 \mathrm{~Hz}$. This structure borne noise problem is observed to be in a specific frequency 
band from $190 \mathrm{~Hz}$ to $200 \mathrm{~Hz}$ also in other vehicles with different suspension assembly systems [12].

In order to reduce the vibrational energy entering the vehicle body through the suspension, the SUV is equipped with a TVA on each of the rear shock towers. Each of the TVAs weighs $1.46 \mathrm{~kg}$ and it is mounted on the top of the shock towers, as shown in Figure 1. These TVAs are designed to be effective around $190 \mathrm{~Hz}$.

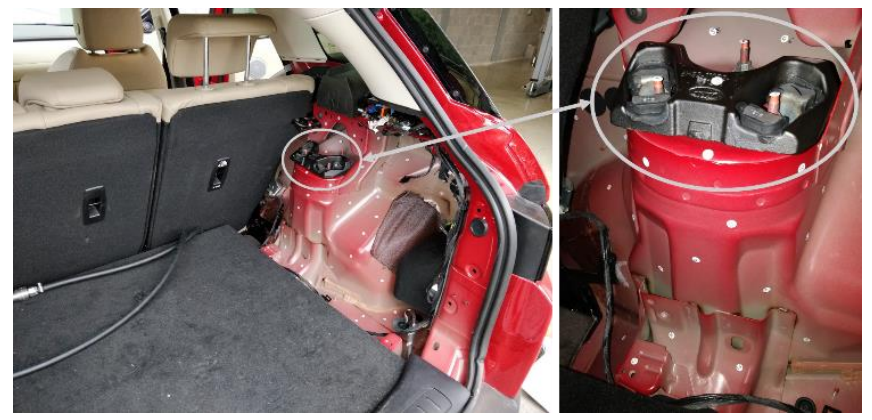

Figure 1 - Right rear shock tower of the vehicle with the TVA installed, highlighted by the gray circles.

To investigate the performance of the metamaterial concept and to compare it with the current NVH solution, the TVA is replaced by patches composed of resonant elements. The patches are added around the areas where the shock absorbers are connected to the shock tower, indicated by the added white paper tape in Figure 2.
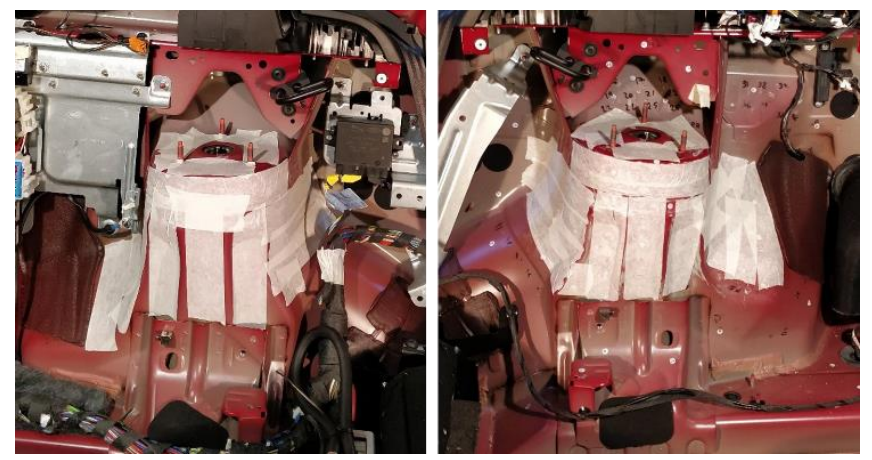

Figure 2 - The areas to be treated by the patches, marked with white paper tape: (left) left rear shock tower, (right) right rear shock tower.

\section{Metamaterial solution design}

Locally resonant metamaterials exhibit stop band behavior when two conditions are met:

1. The net sum of the forces on the host structure exerted by the resonant elements needs to be non-zero [13].

2. The resonant elements of the metamaterial system are added onto the host structure on subwavelength scale with respect to the structural wavelength that propagates in the host structure at the designed frequency [14].

Since these two conditions can be satisfied independently of the host structure through (1) the design and (2) the relative position of the resonant elements in the host structure, then stop band behavior can be also achieved in the rear shock towers.

Page 2 of 9
To comply with the first condition, the resonant element is designed to resemble a mass-spring-damper system consisting of two cantilever beams with an end point mass on each end. A support connects the two beams and the base, the latter is the connection area between the resonant element and the vehicle (Figure 3). The design of the resonant element follows the same philosophy as previous work of the authors $[5,6,8,9]$. This design allows to have a clear low-frequent bending mode that introduces localized resonant behavior in the host structure [5]. Moreover, due to its simple shape it allows a straightforward tailoring of the resonance frequency of the first bending mode by changing the dimensions of the end masses and the thickness of the beams. The resonant elements are made of polyamide and they are realized by selective laser sintering.
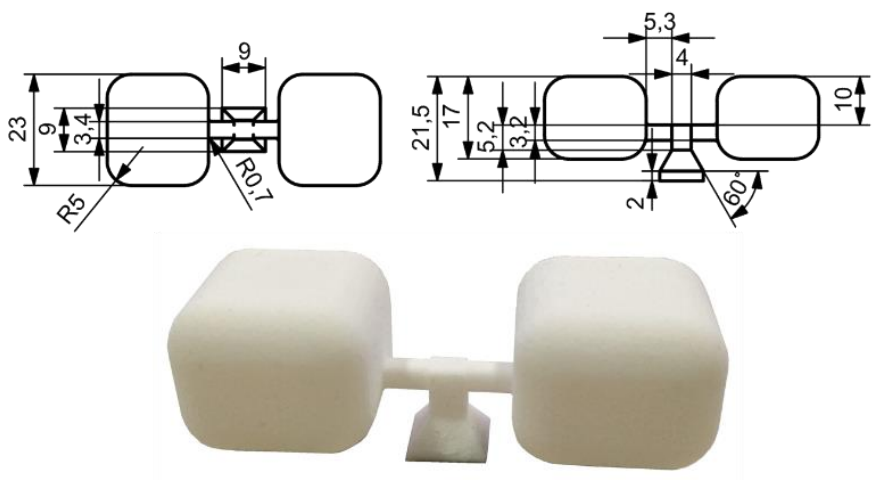

Figure 3 - (Top) Design of the manufactured resonant element. The dimensions are given in $\mathrm{mm}$. (Bottom) Picture of the manufactured resonant element.

The resonant elements are designed to be effective in a frequency range around $190 \mathrm{~Hz}$, similar as for the TVA. They are designed to have at the same resonance frequency the first in-plane and out-of-plane bending mode, in which the two masses move in-phase (Figure 4). The geometrical dimensions of the resonant element are updated, ending up to a resonance frequency of $193 \mathrm{~Hz}$, which is close enough to the target frequency range of $190 \mathrm{~Hz}$. The highest displacement of the masses at the designed resonance frequency is along the y direction for the in-plane mode and the $\mathrm{z}$ direction for the out-of-plane mode.
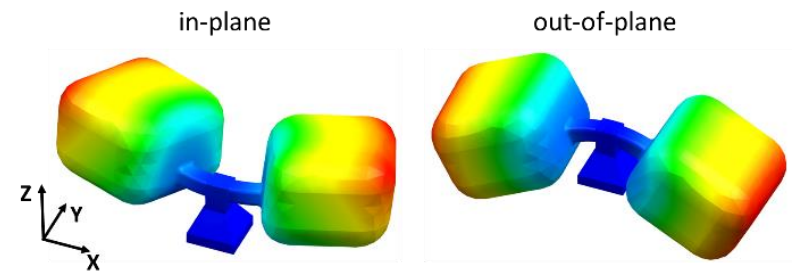

Figure 4-Mode shapes of the first bending mode in in-plane and outof-plane direction at $193 \mathrm{~Hz}$.

The resonant elements are designed using a Finite Element (FE) approach. In this model, the material properties of polyamide, which are reported in Table 1, are used. The FE model consists of 6807 linear solid elements and the base of the resonant element is modelled as clamped. To verify the design, the resonance frequencies of 8 printed resonant elements are measured. The experimental procedure consists of gluing the base of the manufacture resonant elements on an electrodynamic shaker and measure the velocity on the tip of the end mass with a Scanning Laser Doppler Vibrometer Polytec PSV-500. A Root Mean Square (RMS) average of the resonance frequencies of the 
8 different samples is evaluated. The difference between the resonance frequency numerically predicted and experimentally measured is less than $1 \%$.

Table 1 - Material parameters of the polyamide material of the resonant elements and of the steel of the host structure.

\begin{tabular}{|l|l|l|}
\hline & \multicolumn{1}{|c|}{ Polyamide } & \multicolumn{1}{c|}{ Steel } \\
\hline Young's modulus & $2.05 \mathrm{GPa}$ & $210 \mathrm{GPa}$ \\
\hline Poisson's ratio & 0.4 & 0.3 \\
\hline Density & $965 \mathrm{~kg} / \mathrm{m}^{3}$ & $7800 \mathrm{~kg} / \mathrm{m}^{3}$ \\
\hline Structural damping & $4 \%$ & $1 \%$ \\
\hline
\end{tabular}

\section{Design and position of the resonant elements on the host structure}

In order to facilitate the installation of the resonant elements on the rear shock towers of the vehicle the patches are made in a similar form as the shock tower. To ease installation four patches are made for each shock tower instead of one.

The patches are designed with the aid of a CAD model of the rear shock towers: the resonant elements are added on the shock tower around the connections of the shock absorber, as shown in Figure 2. This location was chosen since it is expected that this area is part of the transfer path for the vibration energy coming from the tires and travelling into the body. From previous studies it is known that a minimum amount of resonant elements are required to prevent energy from propagating through a structure [9]. Therefore, the dimension of the treated area is chosen as a compromise between occupying the least space required and still host a minimum of 5 rows of resonators, counted from the closest resonant element to the force input location to the farthest. The chosen lay-out results in a total of 105 resonant elements, distributed on both the rear shock towers. Since the geometry of the rear shock towers is not symmetric, the right shock tower has space to host three resonant elements more than the left shock tower.

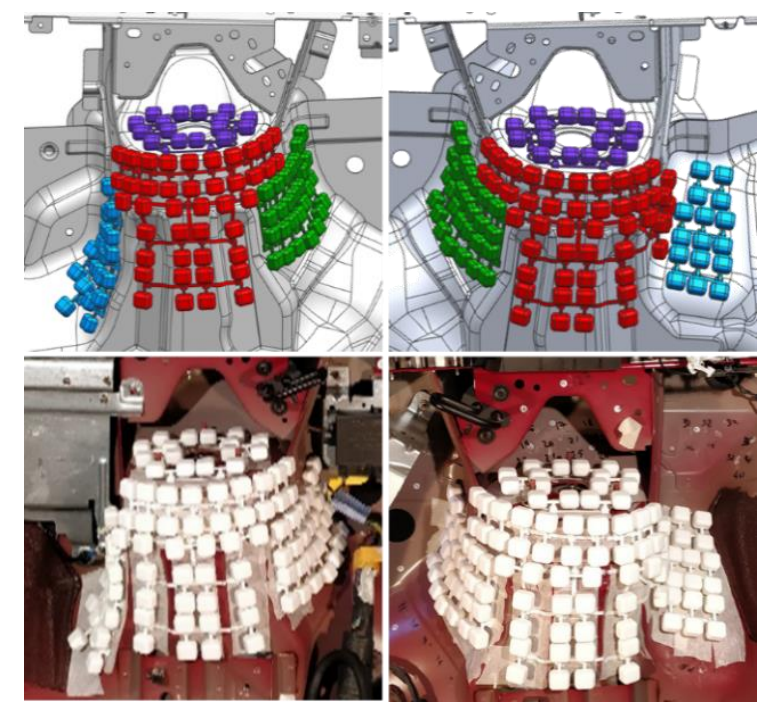

Figure 5-(Top) CAD and (bottom) picture of the installation in the vehicle of the patches on the rear shock towers. Left and right figures represent the left and the right shock tower, respectively.

Page 3 of 9
The resonant elements are added onto the host structure on a more or less regular grid of dimensions $65 \mathrm{~mm}$ in $\mathrm{x}$ direction and $25 \mathrm{~mm}$ in y direction, according to the reference system in Figure 4. These dimensions allow the resonators to be added on a subwavelength scale and thus to satisfy the second condition for achieving stop band behavior. The bases of the resonant elements are connected together by beams with rectangular cross-sections, $1 \mathrm{~mm}$ thick and $4 \mathrm{~mm}$ wide. These dimensions allow the connections to be flexible enough to ensure a good fit of the patches onto the shock tower, compensating possible geometrical variations due to manufacturing for both the patches and the shock tower. The patches are connected to the shock tower with superglue. Figure 5 shows the position of the patches in the CAD model and in the vehicle. The colors red, purple, green and blue in the CAD model indicate the four different patches. In total the two patches together weigh $1.52 \mathrm{~kg}$, reducing the added mass with respect to the current $\mathrm{NVH}$ solution by $48 \%$.

\section{Numerical prediction of the stop band behavior}

To predict stop band behavior in the metamaterial system, Unit Cell (UC) modelling is used. This numerical method makes use of infinite periodic structure theory and the Bloch-Floquet theorem [15] to describe, in a computationally fast and cheap way, the response of an infinite periodic metamaterial system in terms of a UC, which results in an eigenvalue problem. Stop band behavior in the infinite periodic metamaterial system corresponds to a frequency range of vibration attenuation in the finite metamaterial system.

A flat plate of $2 \mathrm{~mm}$ thickness describes the UC of the shock tower. For modelling purpose, the resonant elements are added onto the host structure on a periodic grid of dimensions $65 \times 25 \mathrm{~mm}$, although stop band behavior in locally resonant metamaterials do not rely on periodicity [14]. These dimensions define the geometry of the UC of the metamaterial system analyzed.

For the sake of this analysis, although the real shock tower resembles a curved shell, describing the UC of the shock tower as a flat plate rather than a curved plate is preferred for the following reasons:

- whenever waves propagate in the system, the stop band mechanism can be achieved for those waves independently from the host structure geometry [16];

- similar stop band behavior within reasonable approximations is achieved in metamaterial systems with flat and curved host structure [16];

- $\quad$ stop band behavior is easier to be analyzed due to the simpler dispersion properties.

An undamped FE model of the UC with periodic Bloch-Floquet boundary conditions is applied $[17,18,19]$. The FE model of the UC is, regarding the resonant element, the same as the FE model described in previous section; for the host structure, 429 linear shell elements are used. The assumed material properties for steel, used to model the host structure, are reported in table 1.

The UC analyzed, the Irreducible Brillouin Contour (IBC) [15] chosen according to [20] and the dispersion diagram are reported in Figure 6. Stop bands are identified in the dispersion diagram as the frequency regions where no solution is found.

In the dispersion diagrams in Figure 6 three wave types are identified: bending (B), shear (S) and longitudinal (L). A distinction among the curves in the dispersion diagrams is made based on percentage of the out-of-plane versus the in-plane motion of the host structure. The 
waves with a pure in-plane and pure out-of-plane motion are indicated in the dispersion diagrams by the dark blue and dark red color respectively. Other colors indicate waves with a hybrid in-plane and out-of-plane motion.

Comparing the dispersion diagram of the UC of the host structure only with the dispersion diagram of the metamaterial UC, it can be observed for out-of-plane waves that the bending wave type (B), in Figure 6 splits in two branches, (B' and B"), achieving a complete stop band from $193.1 \mathrm{~Hz}$ to $224.1 \mathrm{~Hz}$ along the IBC. The situation is different for the in-plane waves: the shear wave type (S) splits also in two branches (S' and S"), achieving a directional stop band, from $193 \mathrm{~Hz}$ to $224 \mathrm{~Hz}$, along the $d_{1}$ direction of the IBC. For the same $d_{1}$ direction, a stop band is not achieved for longitudinal wave type $(\mathrm{L})$ in the same frequency range, due to the continuous branch. Along the $\mathrm{d}_{2}$ direction of the IBC, a directional stop band is achieved for the longitudinal wave type (L) that splits in two branches (L' and L") and not for shear wave type (S). These effects are due to the direction of the motion of the in-plane and out-of-plane bending modes of the resonant element, described in the previous section. The out-of-plane mode of the resonant element always exerts a non-zero net force on the host structure, independently from the propagation direction of the propagating out-of-plane wave. The in-plane mode, instead, only exerts a non-zero net force in $\mathrm{d}_{2}$ direction. Therefore, stop band exists for those waves that have a displacement component parallel to $\mathrm{d}_{2}$ direction.
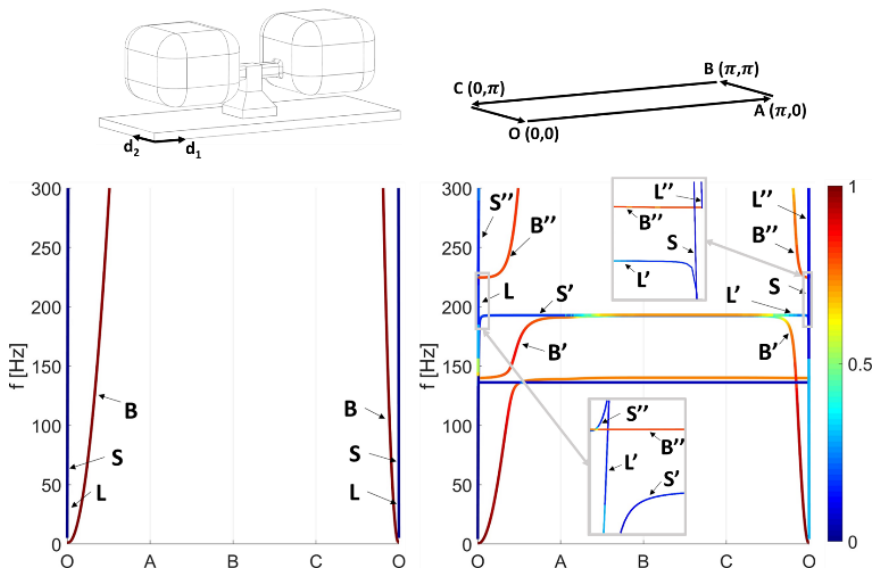

Figure 6-(Top) Sketch of the UC consisting of the host structure and a resonant element. The coordinates of the propagation directions $O, A, B, C, O$ are indicated. (Bottom left) Dispersion diagram for the UC, consisting of the host structure only. (Bottom right) Dispersion diagram for the metamaterial UC. For both graphs, the IBC is indicated on the horizontal axis. The vertical axis represents the frequency. The letters $(B),(S)$ and $(L)$ and the color map indicate the type of waves propagating in the structure.

When damping is introduced in the metamaterial system, decaying waves exist in the stop band. Therefore, for real metamaterial system where the damping is always present, the stop band becomes a zone of strongly attenuated wave propagation [14, 21].

Wave dispersion in systems with complex geometry as the shock tower is characterized by a highly coupled nature of the propagative waves. These waves convert to different wave types at junctions of flat or curved plates with different angles [22]. In order to minimize the transmission of energy along the treated transfer paths, whether it is carried by out-of-plane or in-plane waves, the proposed metamaterial design attenuates the propagation of more than one wave type per propagation direction.

Page 4 of 9

\section{Test procedure}

This section describes the test procedure used for evaluating the performance of the metamaterial concept proposed with respect to the current TVA solution. Two different tests are performed: in-lab to have a controlled excitation and on-road to assess actual performance in operation conditions. The measurement setup and the test procedure for the two different tests are explained separately. In these tests, only frequency responses are considered, corresponding to steady state conditions. Signals in time domain, e.g. for shock response, were not investigated.

\section{In-lab tests}

The in-lab tests allow to have a controlled excitation of the entire system. To this end, two electrodynamic shakers (the Modal Shop $2100 \mathrm{E} 11)$ are installed under the vehicle and connected to the bottom of the rear suspensions through a stinger and an impedance head (PCB Model 288D01). Figure 7 shows the setup of the shaker on the rear right suspension.

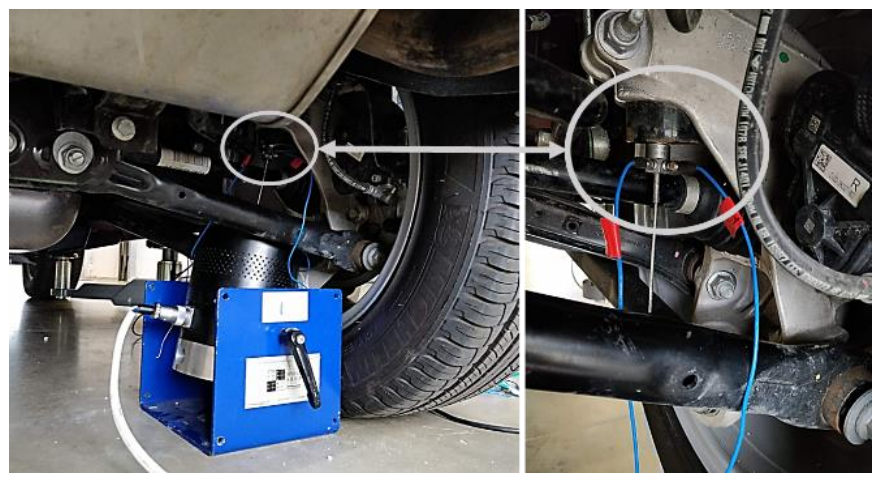

Figure 7 - Setup of the shaker with stinger and impedance head connected under the rear right suspension of the vehicle.

A random white noise signal is applied as excitation to the shaker. This excitation allows to evaluate the performance of the $\mathrm{NVH}$ solutions under steady state conditions, in order to mimic a regular road profile. The excitation frequency range is from $20 \mathrm{~Hz}$ to $512 \mathrm{~Hz}$ with $0.5 \mathrm{~Hz}$ of resolution.

Sound Pressure Level (SPL) is measured in the interior compartment by means of 3 microphones (PCB Model 378B02). The microphones are located in the following positions, as sketched in Figure 8:

$$
\begin{array}{ll}
\text { - } & \text { Driver right ear (D) } \\
\text { - } & \text { Front passenger left ear (FP) } \\
\text { - } & \text { Rear right passenger left ear (RP) }
\end{array}
$$

These microphone positions are chosen for the following reasons:

- To have an adequate distribution of the sensors to measure the noise in the interior compartment perceived by the driver and the passengers;

- To minimize the influence of other noise sources, e.g. windows, by measuring close to the longitudinal axis of the vehicle. 
The microphones are secured between the head restraints and the seat back. Figure 8 shows the positions of the microphones on the seats of the driver and front passenger.

For the tests the tire pressure is set at $2.1 \mathrm{~atm}$ and the temperature of the air in the interior compartment is $20{ }^{\circ} \mathrm{C}$.

Frequency Response Functions (FRFs) pressure/force are used to evaluate the response of the system. Fifty averages are taken to acquire FRFs with a coherence above 0.9 in the frequency range of interest.
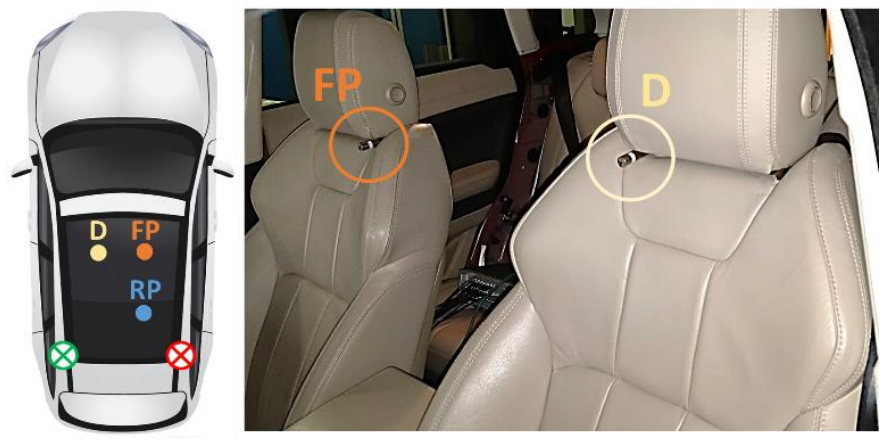

Figure 8 - Sketch of the microphone positions in the interior compartment and on the seats for the driver and front passenger (shaded circles) and locations of the excitation under the rear suspensions (circles with crosses): the left and the right shaker are indicated in green and red, respectively [23].

\section{On-road tests}

The on-road tests allow to evaluate the performance of the NVH solutions under operation conditions. Two different road profiles and driving conditions are chosen and they are summarized in Table 2.

Table 2 - Summary of the road profile and driving speed of the onroad tests. The maximum speed tolerance is indicated.

\begin{tabular}{|l|l|}
\hline Road profile & Speed \\
\hline road-1: asphalt & $50 \mathrm{kph} \pm 2 \mathrm{kph}$ \\
\hline road-2: cobblestones & $20 \mathrm{kph} \pm 1 \mathrm{kph}$ \\
\hline
\end{tabular}

The on-road tests are made on both smooth (road-1) and rough surface (road-2). The on-road tests are performed on dry public roads with low traffic such that constant speed could be maintained and no pass-bynoise influence from other vehicles occurred during the acquisitions. The two driving speeds are chosen to be within the maximum speed allowed in the public roads used for the tests. The driving speed is maintained constant within a tolerance of $\pm 5 \%$, as reported in Table 2. Same tire pressure level and interior compartment temperature, as for the in-lab tests, are set.

The excitation level, at which the vehicle is subjected during the driving tests, is measured by two tri-axial accelerometers (PCB Model 356A15), installed at the location of the impedance heads used in the in-lab tests. The SPL is measured in the interior compartment with the same setup used for the in-lab tests.
For these tests, the Power Spectral Densities (PSDs) of the pressure and of the acceleration are evaluated. In order to obtain a converged spectrum profile, 150 averages were required during each test. Each measurement is repeated 5 times for the different road profiles and driving speeds. For each sensor, a Root Mean Square (RMS) of the PSD across the measurements is calculated as follows:

$R M S_{P S D_{x}}(\omega)=\sqrt{\frac{1}{N} \sum_{i=1}^{N}\left|P S D_{x}(\omega)\right|^{2}}$.

In equation (2), $\omega$ is the frequency, $N$ is the number of measurements for that driving condition and the $x$ indicates the quantity measured by the sensor (pressure or acceleration). For the evaluation of the threeaxial accelerometers, an additional post-processing is required after applying equation (2): the value of the RMS PSD acceleration in three directions combined is calculated for each accelerometer based on the signals of the three accelerometer directions, as follows:

$R M S_{P S D_{a c c}}^{\text {tot }}(\omega)=\sqrt{\sum_{i=1}^{3}\left(R M S_{P S D_{a c c}}^{i}(\omega)\right)^{2}}$,

where the $i$ in the sum indicates the different accelerometer directions.

\section{Test results and discussion}

In this section the test results for the different configurations measured are shown and discussed. An overview of the measured configurations is reported in Table 3.

Table 3 - Summary of the configurations tested.

\begin{tabular}{|l|l|}
\hline Configuration name & Description \\
\hline Bare & $\begin{array}{l}\text { No NVH treatment installed } \\
\text { on the rear shock towers }\end{array}$ \\
\hline TVA & $\begin{array}{l}\text { The TVAs are installed on } \\
\text { the rear shock towers }\end{array}$ \\
\hline Metamaterial & $\begin{array}{l}\text { The TVAs are replaced by } \\
\text { the patches }\end{array}$ \\
\hline
\end{tabular}

The in-lab test results and the on road test results are presented in consecutive subsections. To better compare the NVH performance of the three configurations in the frequency range of interest, the graphs are shown from $100 \mathrm{~Hz}$ to $300 \mathrm{~Hz}$. All the SPL results are shown in $\mathrm{dBA}$ and the $\mathrm{dB}$ reference value is $20 \mathrm{e}-6 \mathrm{~Pa}$.

\section{In-lab test results}

The FRF SPL results for the three configurations tested are shown separately for the source of excitation acting on the rear left shock tower (Figure 9) and on the rear right shock tower (Figure 10).

From the results, it can be observed that the bare configuration has a peak in the SPL around $195 \mathrm{~Hz}$. The TVA configuration has a lower SPL than the bare configuration in the range from $185 \mathrm{~Hz}$ to $210 \mathrm{~Hz}$ for all the microphone positions, except for the rear passenger in the case that the excitation is on the left shock tower. For the latter case, however, there is not a clear peak around $195 \mathrm{~Hz}$. Outside this frequency range, the SPL measured for the bare and TVA configuration are similar for all cases. The metamaterial configuration reduces the SPL in the interior compartment, similar as for the TVA configuration. It can be observed that, for excitation on the left shock 
tower, the metamaterial configuration performs similar as the TVA configuration for the frequency range from $185 \mathrm{~Hz}$ to $200 \mathrm{~Hz}$. However, from $200 \mathrm{~Hz}$ to $210 \mathrm{~Hz}$, the metamaterial configuration performs worse than the TVA. For the excitation on the right shock tower, the metamaterial configuration performs similar as for the TVA configuration in the same frequency range, from $185 \mathrm{~Hz}$ to $210 \mathrm{~Hz}$.
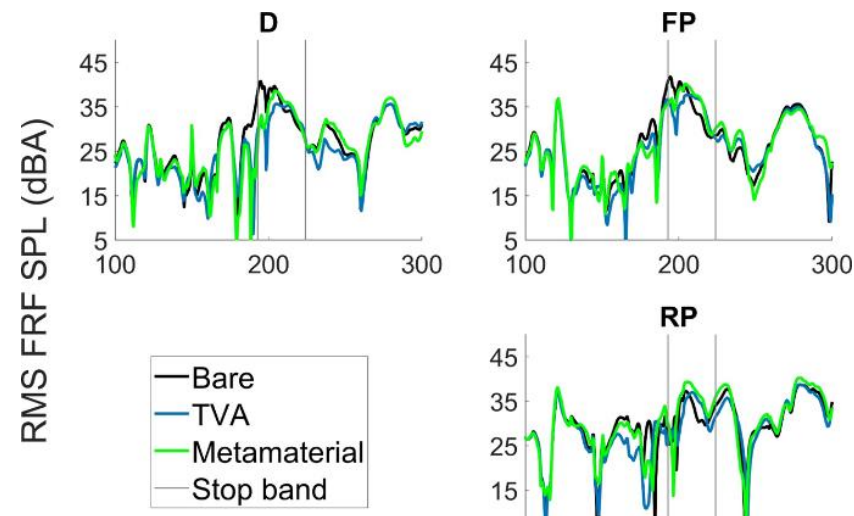

Frequency $(\mathrm{Hz})$

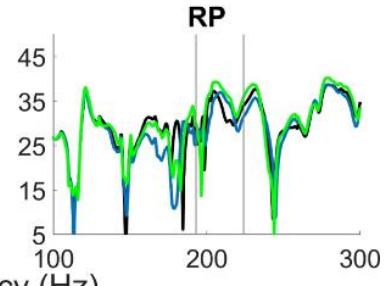

Figure 9 - RMS FRF SPL comparison between the three tested configurations, when the source of excitation is under the rear left suspension. The gray vertical lines indicate the predicted stop band frequency range.
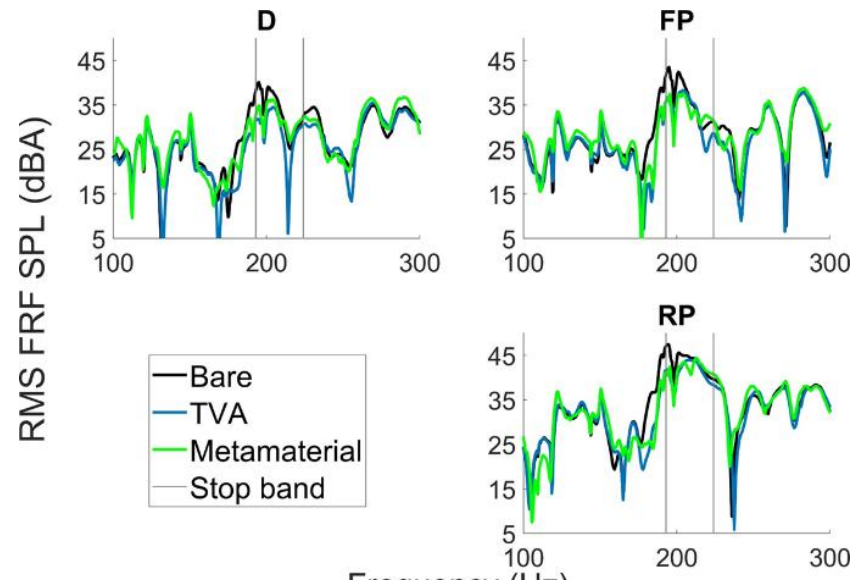

Frequency $(\mathrm{Hz})$

Figure 10 - RMS FRF SPL comparison among the three tested configurations, when the source of excitation is the under the rear right suspension. The gray vertical lines indicate the predicted stop band frequency range.

The metamaterial configuration shows a strong effect in the SPL reduction right before and at the lower bound of the predicted stop band. This effect can be attributed to the variation of wave attenuation throughout the stop band frequency range. Close to the lower bound of the stop band, at the resonance frequency of the resonant element, the wave attenuation is maximum and it decreases towards the upper bound of the stop band [14, 21]. Therefore, the energy injected into the vehicle body is strongly attenuated throughout all the stop band frequency range, but achieving a maximum attenuation at the lower bound.

\section{On-road tests}

Page 6 of 9
In this section, first the results for road-1 are presented and discussed, followed by the results for road-2.

\section{Road-1: asphalt}

The RMS PSD acceleration in Figure 11, measured at the bottom of the rear suspensions for all the tested configurations, indicates that the excitation level for all configuration is similar. Moreover, the maximum standard deviation calculated on the RMS PSD SPL in Figure 12 is within $\pm 1.01 \mathrm{dBA}$, which confirms the consistency of the results between different measurements of the same configuration.

For the PSD acceleration in Figure 11, two sharp peaks can be observed, of which the highest is at $198 \mathrm{~Hz}$. These peaks are also present in the RMS PSD SPL in the interior compartment, as shown in Figure 12. In contrast to the SPL measured in the in-lab tests, the SPL spectrum for the on-road tests shows a more pronounced peak.
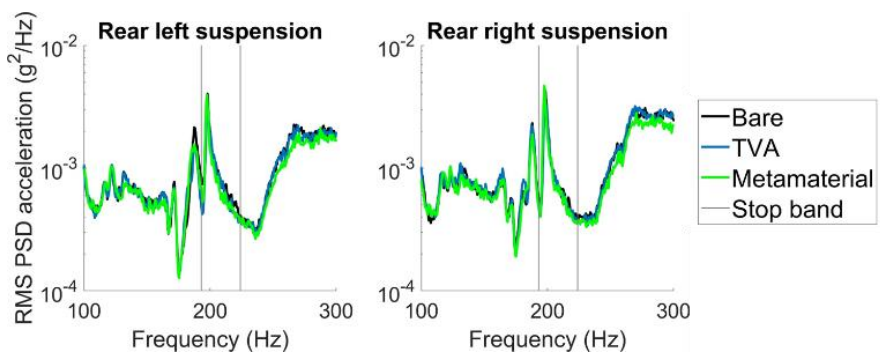

Figure 11 - Comparison of the RMS PSD acceleration measured at the bottom of the rear left and right suspensions for the three tested configurations for the case road-1.

Both the TVA and the metamaterial configuration reduce the SPL peak at $198 \mathrm{~Hz}$. Similar as for the in-lab tests, there is an improvement of the SPL, with respect to the bare configuration, starting from around $185 \mathrm{~Hz}$ for both TVA and metamaterial configurations. Before $185 \mathrm{~Hz}$ and after $210 \mathrm{~Hz}$, the SPL of the three configurations is comparable.

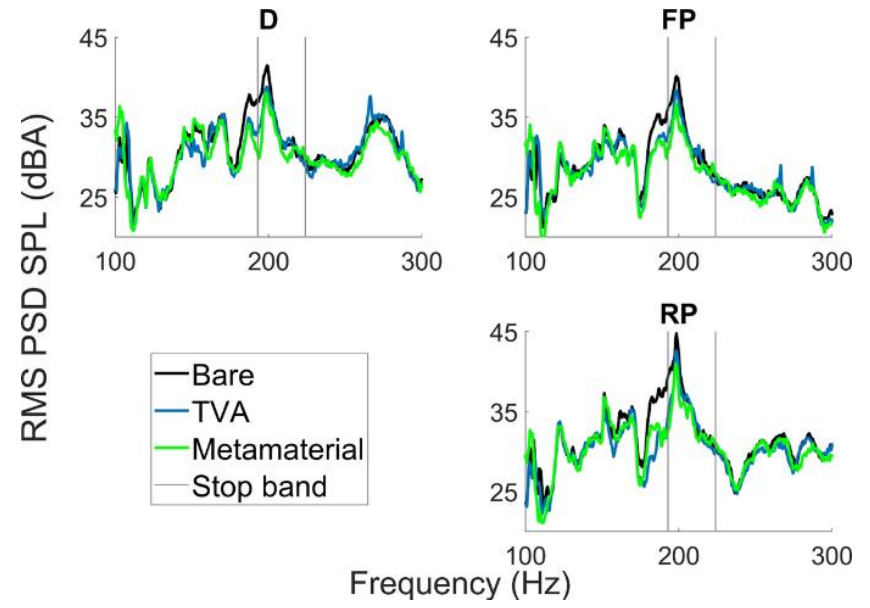

Figure 12 - RMS PSD SPL comparison among the three tested configurations for the case road-1.

At the highest SPL peak at $198 \mathrm{~Hz}$, the metamaterial shows to perform better than the TVA configuration. Table 4 summarizes the SPL values of the three configurations at $198 \mathrm{~Hz}$. 
Table 4 -SPL values at $198 \mathrm{~Hz}$ for the three configurations tested for the case road-1. The values are expressed in $d B A$ and the standard deviation between the 5 different measurements is indicated.

\begin{tabular}{|c|c|c|c|}
\hline Configuration & D (dBA) & FP (dBA) & RP (dBA) \\
\hline Bare & $41.4 \pm 0.80$ & $40.3 \pm 0.84$ & $44.7 \pm 0.85$ \\
\hline TVA & $38.8 \pm 0.79$ & $38.3 \pm 0.73$ & $42.6 \pm 0.65$ \\
\hline Metamaterial & $37.8 \pm 0.70$ & $36.6 \pm 0.71$ & $40.9 \pm 0.84$ \\
\hline
\end{tabular}

\section{Road-2: cobblestone}

Similarly as for the tests on road-1, the tests on road-2 show a comparable excitation level between the three tested configurations, as can be observed for the RMS PSD acceleration in Figure 13. The consistency among the 5 different measurements of the same configuration is confirmed by a maximum standard deviation of \pm 1.07 dBA measured on the PSD SPL, which is shown in Figure 14.

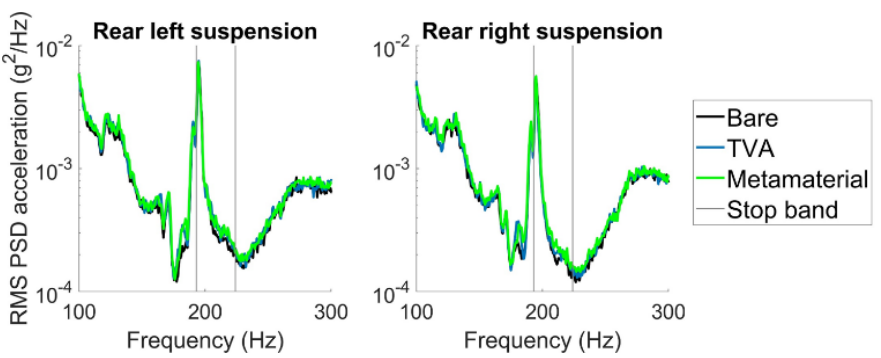

Figure 13 - Comparison of the absolute level of the RMS PSD acceleration measured at the bottom of the rear left and right suspensions for the three tested configurations for the case road-2.

As for the tests on road-1, a sharp peak is measured in the PSD acceleration. The peak occurs at $195 \mathrm{~Hz}$, instead of at $198 \mathrm{~Hz}$ as measured on road-1. At the same frequency, a peak is also measured in the PSD SPL, as shown in Figure 14.
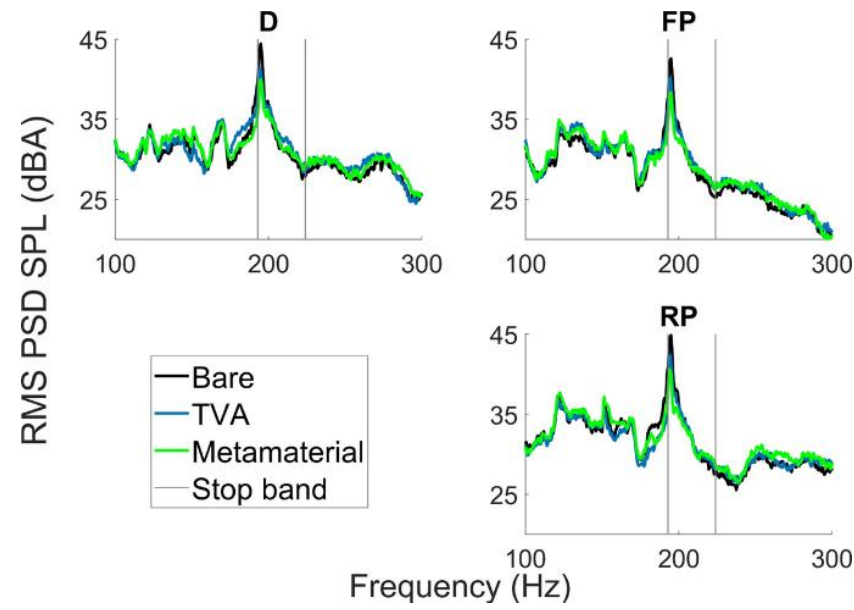

Figure 14 - RMS PSD SPL comparison among the three tested configurations for road-2.

Page 7 of 9

Frequency $(\mathrm{Hz}$
The performance of the TVA and metamaterial configurations for the tests on road-2 is similar as for the tests on road-1. The values of the SPL at $195 \mathrm{~Hz}$ for the three tested configurations are summarized in Table 5.

Table 5 -SPL values at $195 \mathrm{~Hz}$ for the three configurations tested on road-2. The values are expressed in $\mathrm{dBA}$ and the standard deviation between the 5 different measurements is indicated.

\begin{tabular}{|c|c|c|c|}
\hline Configuration & D (dBA) & FP (dBA) & RP (dBA) \\
\hline Bare & $44.4 \pm 1.07$ & $42.6 \pm 0.99$ & $44.9 \pm 1.01$ \\
\hline TVA & $41.1 \pm 0.78$ & $40.1 \pm 0.69$ & $42.3 \pm 0.71$ \\
\hline Metamaterial & $39.4 \pm 0.69$ & $38.2 \pm 0.84$ & $39.9 \pm 1.02$ \\
\hline
\end{tabular}

\section{Summary/Conclusions}

This paper applies the concept of metamaterials to reduce the noise in the interior compartment of a vehicle, caused by tire/road interaction while driving, and leading to vibrational energy being injected into the vehicle body through the suspension assembly system. The challenge in the application is to replace the current NVH solution, a TVA installed on each of the rear shock towers, with a lighter metamaterial solution and yet maintaining similar $\mathrm{NVH}$ performance in this hard-toaddress low frequency region.

The metamaterial system is realized by installing patches onto the rear shock towers. The patches are composed by resonant elements and they are realized by selective laser sintering. The resonant elements are modeled in FE and their performance are predicted with the UC modelling approach. A flat plate with a resonant element describes the UC of the shock tower. The numerical analysis provides the frequency range where stop band behavior is achieved for an infinite periodic metamaterial system, which corresponds, within reasonable approximations, to the frequency range of vibration attenuation in the real shock towers.

Three configurations are tested and compared: bare, TVA and metamaterial. Tests are made in-lab using a controlled excitation and on-road in operation conditions, both on an asphalt and a cobblestone road. In all cases, SPL levels at driver and passengers' ear locations are evaluated, resulting in following conclusions:

- $\quad$ Both the TVA and the metamaterial solution show to reduce the SPL inside the interior compartment between 185 and $200 \mathrm{~Hz}$.

- In-lab tests: the TVA and the metamaterial configurations have similar performance from $185 \mathrm{~Hz}$ to $200 \mathrm{~Hz}$ and they both outperform the bare case. After $200 \mathrm{~Hz}$, the metamaterial configuration has similar SPL performance as for the bare configuration, while the TVA configuration outperforms the bare case until $210 \mathrm{~Hz}$. After this frequency, the three solutions have similar SPL levels.

On-road tests: the problematic frequency region is more apparent due to a sharp peak appearing in the SPLs around $190-200 \mathrm{~Hz}$. Both TVA and metamaterial configurations show to reduce the peak in SPL. The metamaterial configuration performs better than the TVA configuration. 
- $\quad$ Both for in-lab and on-road tests, the highest SPL reduction in the interior compartment for the metamaterial solution occurs at frequencies closer to the lower bound of the predicted stop band.

Test results verify that the metamaterial configuration has similar and/or better NVH performance than the TVA configuration, while reducing the added mass of the current NVH solution by $48 \%$.

Although the approximations made in regard to the metamaterial design and the stop band behavior analysis were reasonable, in presence of a full FE model of the vehicle the results, approximations or the metamaterial design might change.

\section{References}

1. Mallick, P. K., (2010). Materials, design and manufacturing for lightweight vehicles. Elsevier. doi.org/10.1533/9781845697822

2. Fritschi, L., Brown, A., Kim, R., Schwela, D., Kephalopolous, S., (2011). Burden of disease from environmental noise: quantification of healthy life years lost in Europe. World Health Organization, Regional Office for Europe, Copenhagen. ISBN: 9789289002295

3. Fahy, F. J., Gardonio, P., (2007). Sound and structural vibration: radiation, transmission and response. Elsevier. doi.org/10.1016/b9780-12-373633-8.x5000-5

4. Liu, Z., Zhang, X., Mao, Y., Zhu, Y., Yang, Z., Chan, C., . . Sheng, P., (2000). Locally resonant sonic materials. Science, 289(5485), 1734--1736. doi:10.1126/science.289.5485.1734

5. Claeys, C. C., Deckers, E., Pluymers, B., \& Desmet, W. (2016). A lightweight vibro-acoustic metamaterial demonstrator: numerical and experimental investigation. Mechanical Systems and Signal Processing, 70, 853--880. doi:10.1016/j.ymssp.2015.08.029

6. De Melo Filho, N. G. R., Van Belle, L., Claeys, C., Deckers, E., Desmet, W., (2019). Dynamic mass based sound transmission loss prediction of vibro-acoustic metamaterials double panels applied to the mass-air-mass resonance. Journal of Sound and Vibration, 442, 28--44. doi.org/10.1016/j.jsv.2018.10.047

7. Van Belle, L., Claeys, C., Deckers, E., Desmet, W., (2018). Influence of damping on the sound insulation of infinite and finite locally resonant metamaterial plates. Proceeding of ISMA 2018, KU Leuven.

8. Claeys, C., Melo, N. F., Van Belle, L., Deckers, E., \& Desmet, W., (2017). Design and validation of metamaterials for multiple structural stop bands in waveguides. Extreme Mechanics Letters, 12, 7--22. doi:10.1016/j.eml.2016.08.005

9. Sangiuliano, L., Claeys, C., Deckers, E., Pluymers, B., Desmet, W., (2018). Force isolation by locally resonant metamaterials to reduce NVH. SAE Technical Paper Series. doi.org/10.4271/2018-01-1544

10. Douville, H., Masson, P., Berry, A., (2006). On-resonance transmissibility methodology for quantifying the structure-borne road noise of an automotive suspension assembly. Applied Acoustic 67, 358--382. doi: 10.1016/j.apacoust.2005.07.002

11. Kindt, P., Berckmans, D., De Coninck, F., Sas P., Desmet, W., (2009). Experimental analysis of the structure-borne tyre/road noise due to road discontinuities. Mechanical Systems and Signal Processing 23, 2557--2574. doi: 10.1016/j.ymssp.2009.04.005

12. Tatlow, J., Ballatore, M., (2017). Road noise input identification for vehicle interior noise by multi-reference Transfer Path Analysis. Procedia engineering 199, 3296--3301. doi: 10.1016/j.proeng.2017.09.412

13. Wang, G., Wen, X., Wen, J., Shao, L., \& Liu, Y., (2004). Twodimensional locally resonant phononic crystals with binary structures. Physical review letters, 93(15), 154302. doi:10.1103/PhysRevLett.93.154302

14. Claeys, C., Vergote, K., Sas, P., \& Desmet, W., (2013). On the potential of tuned resonators to obtain low-frequency vibrational stop bands in periodic panels. Journal of Sound and Vibration, 332(6), 1418--1436. doi:10.1016/j.jsv.2012.09.047

15. Brillouin, L., (1946). Wave propagation in periodic structures (Vol. 2nd). McGraw-Hill Book Company.

16. Nateghi, A., Van Belle, L., Claeys, C., Deckers, E., Pluymers, B., \& Desmet, W. (2017). Wave propagation in locally resonant cylindrically curved metamaterial panels. International Journal of Mechanical Sciences, 127, 73--90. doi: 10.1016/j.ijmecsci.2016.07.003

17. Mace, B. R., \& Manconi, E., (2008). Modelling wave propagation in two-dimensional structures using finite element analysis. Journal of Sound and Vibration, 318(4), 884--902. doi:10.1016/j.jsv.2008.04.039

18. Diaz, A. R., Haddow, A. G., \& Ma, L., (2005). Design of band-gap grid structures. Structural and Multidisciplinary Optimization, 29(6), 418--431. doi:10.1007/s00158-004-0497-6

19. Langley, R. S. (1993), A note on the force boundary conditions for two-dimensional periodic structures with corner freedoms. Journal of Sound and Vibration, 162(2), 377--381. doi: 10.1006/jsvi.1993.1341

20. Maurin, F., Claeys C., Deckers, E., Desmet, W., (2018). Probability that a band-gap extremum is located on the irreducible Brillouin-zone contour for the 17 different plane crystallographic lattices. International Journal of Solids and Structures, 135, 26--36. doi.org/10.1016/j.ijsolstr.2017.11.006

21. Van Belle, L., Claeys, C., Deckers, E., Desmet, W., (2017). On the impact of damping on the dispersion curves of a locally resonant metamaterial: Modelling and experimental validation. Journal of Sound and Vibration, 409, 1--23. doi: 10.1016/j.jsv.2017.07.045

22. Cremer, L., Heckl, M., Petersson, B.A.T., (2005). Structure-borne sound. Springer, 341--359. ISBN: 3-540-22696-6.

23. Sketch top view of car image courtesy of "Designed by Freepik", www.freepik.com.

\section{Contact Information}

luca.sangiuliano@kuleuven.be

\section{Acknowledgments}

The Research Fund KU Leuven is gratefully acknowledged for its support. This research was partially supported by Flanders Make, the Strategic Research Centre for the manufacturing industry. The research of E. Deckers is funded by a grant from the Research Foundation - Flanders (FWO).

\section{Definitions/Abbreviations}

$\begin{array}{ll}\text { B } & \text { Bending wave type } \\ \text { D } & \text { Driver ear position } \\ \text { FE } & \text { Finite Element } \\ \text { FP } & \text { Front Passenger ear position } \\ \text { FRF } & \text { Frequency Response Function } \\ \text { IBC } & \text { Irreducible Brillouin Contour }\end{array}$

$\mathbf{L}$
Longitudinal wave type

Page 8 of 9 
NVH

PSD

RMS

RP
Noise, Vibration Harshness

Power Spectral Density

Root Mean Square

Right Rear Passenger ear position
$\mathbf{S}$

SPL

TVA

UC
Shear wave type

Sound Pressure Level

Tuned Vibration Absorber

Unit Cell

Page 9 of 9 